Supplement of Ocean Sci., 16, 1247-1259, 2020

https://doi.org/10.5194/os-16-1247-2020-supplement

(C) Author(s) 2020. This work is distributed under

the Creative Commons Attribution 4.0 License.

(c) (i)

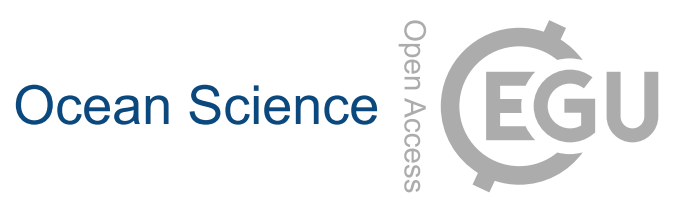

Supplement of

\title{
Changes in detrital sediment supply to the central Yellow Sea since the last deglaciation
}

\author{
Hyo Jin Koo and Hyen Goo Cho \\ Correspondence to: Hyen Goo Cho (hgcho@gnu.ac.kr)
}

The copyright of individual parts of the supplement might differ from the CC BY 4.0 License. 


\section{Core 11YS-PCL14}

\begin{tabular}{|c|c|c|c|c|c|c|c|}
\hline \multirow{2}{*}{ Unit } & \multirow{2}{*}{$\begin{array}{l}\text { depth } \\
(\mathrm{cm})\end{array}$} & \multirow{2}{*}{$\begin{array}{c}\text { Mean grain size } \\
\text { (Phi) }\end{array}$} & \multicolumn{3}{|c|}{ Grain Contents (\%) } & \multicolumn{2}{|c|}{ Clay mineral co } \\
\hline & & & Sand & Silt & Clay & Smectite & Iillite \\
\hline \multirow{10}{*}{ Unit 1} & $\overline{77}$ & 9.5 & 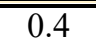 & 27.2 & 72.5 & 1.6 & 66.6 \\
\hline & 47 & 8.31 & 1.0 & 41.1 & 58.0 & 1.3 & 68.2 \\
\hline & 57 & 8.63 & 0.7 & 39.7 & 59.6 & 1.8 & 68.7 \\
\hline & 67 & 8.76 & 0.3 & 35.9 & 63.8 & 2.0 & 68.5 \\
\hline & 77 & 9.26 & 0.3 & 29.6 & 70.1 & 2.3 & 71.8 \\
\hline & 87 & 8.68 & 0.8 & 38.2 & 61.1 & 2.2 & 71.2 \\
\hline & 97 & 8.49 & 0.6 & 42.7 & 56.7 & 2.1 & 68.8 \\
\hline & 107 & 8.71 & 0.8 & 40.8 & 58.4 & 2.1 & 67.8 \\
\hline & 117 & 8.84 & 0.8 & 38.1 & 61.1 & 1.2 & 69.8 \\
\hline & 127 & 8.78 & 0.6 & 39.9 & 59.5 & 2.8 & 67.9 \\
\hline \multirow{8}{*}{ Unit 2-1 } & 137 & 8.35 & 1.8 & 45.9 & 52.3 & 3.2 & 74.7 \\
\hline & 147 & 8.4 & 3.0 & 43.5 & 53.5 & 2.7 & 70.7 \\
\hline & 157 & 7.03 & 17.1 & 48.9 & 34.0 & 3.9 & 69.1 \\
\hline & 167 & 7.51 & 12.8 & 47.5 & 39.7 & 3.9 & 67.5 \\
\hline & 177 & 6.58 & 14.5 & 59.2 & 26.4 & 4.5 & 69.2 \\
\hline & 187 & 6.49 & 19.2 & 56.3 & 24.5 & 4.2 & 69.7 \\
\hline & 197 & 6.1 & 18.6 & 59.5 & 21.9 & 6.3 & 65.9 \\
\hline & 207 & 6.09 & 19.6 & 59.2 & 21.2 & 4.7 & 65.1 \\
\hline \multirow{10}{*}{ Unit 2-2 } & 217 & 5.29 & 44.2 & 41.0 & 14.8 & 4.7 & 66.2 \\
\hline & 227 & 5.87 & 31.1 & 51.2 & 17.8 & 4.5 & 63.4 \\
\hline & 237 & 6.29 & 16.9 & 62.1 & 21.0 & 5.1 & 63.7 \\
\hline & 247 & 6.24 & 18.2 & 61.2 & 20.6 & 5.2 & 65.2 \\
\hline & 257 & 6.26 & 15.6 & 63.7 & 20.7 & 3.6 & 63.4 \\
\hline & 267 & 6.3 & 16.1 & 63.7 & 20.2 & 5.6 & 65.7 \\
\hline & 277 & 6.21 & 15.1 & 65.6 & 19.4 & 6.8 & 64.1 \\
\hline & 287 & 6.03 & 15.7 & 66.5 & 17.8 & 3.9 & 64.1 \\
\hline & 297 & 6.6 & 20.1 & 53.2 & 26.7 & 4.5 & 63.2 \\
\hline & 307 & 6.7 & 12.5 & 61.3 & 26.3 & 3.6 & 64.4 \\
\hline \multirow{25}{*}{ Unit 3} & 317 & 7.38 & 3.8 & 63.4 & 32.8 & 4.7 & 63.2 \\
\hline & 327 & 7.4 & 1.7 & 67.5 & 30.8 & 2.6 & 63.2 \\
\hline & 337 & 7.1 & 1.6 & 69.8 & 28.6 & 4.1 & 61.4 \\
\hline & 347 & 7.03 & 1.8 & 71.3 & 26.9 & 3.8 & 64.2 \\
\hline & 357 & 7.08 & 2.7 & 69.4 & 27.9 & 4.2 & 64.3 \\
\hline & 367 & 7.17 & 0.8 & 70.0 & 29.2 & 3.4 & 60.8 \\
\hline & 377 & 7.37 & 0.7 & 68.7 & 30.6 & 3.7 & 63.1 \\
\hline & 387 & 7.15 & 1.0 & 70.3 & 28.7 & 3.0 & 62.8 \\
\hline & 397 & 7.74 & 1.2 & 60.9 & 38.0 & 2.6 & 63.7 \\
\hline & 407 & 7.23 & 1.6 & 70.9 & 27.5 & 3.5 & 63.4 \\
\hline & 417 & 7.79 & 0.5 & 62.5 & 37.0 & 3.4 & 63.9 \\
\hline & 427 & 8.04 & 0.7 & 58.5 & 40.8 & 3.3 & 62.3 \\
\hline & 437 & 7.85 & 0.2 & 64.2 & 35.6 & 3.4 & 63.4 \\
\hline & 447 & 7.75 & 0.5 & 66.5 & 33.0 & 4.4 & 60.1 \\
\hline & 457 & 7.82 & 0.3 & 65.0 & 34.7 & 3.6 & 62.3 \\
\hline & 467 & 7.38 & 0.5 & 73.1 & 26.4 & 4.0 & 62.0 \\
\hline & 477 & 7.29 & 0.3 & 76.5 & 23.3 & 3.5 & 62.6 \\
\hline & 487 & 7.83 & 0.4 & 63.3 & 36.4 & 3.5 & 63.7 \\
\hline & 497 & 6.88 & 0.4 & 81.5 & 18.1 & 4.2 & 62.9 \\
\hline & 507 & 7.08 & 0.9 & 79.1 & 20.0 & 3.5 & 64.4 \\
\hline & 517 & 6.94 & 1.1 & 81.7 & 17.2 & 2.8 & 63.0 \\
\hline & 527 & 5.09 & 57.8 & 27.8 & 14.4 & 3.9 & 61.2 \\
\hline & 537 & 6.27 & 14.6 & 76.9 & 8.6 & 3.3 & 62.3 \\
\hline & 547 & 6.76 & 8.2 & 90.4 & 1.4 & 3.2 & 62.2 \\
\hline & 557 & 6.12 & 30.0 & 64.6 & 5.4 & 3.8 & 64.0 \\
\hline
\end{tabular}




\begin{tabular}{cccccccc} 
& 567 & 6.14 & 28.7 & 51.7 & 19.6 & 4.9 & 64.1 \\
& 577 & 5.64 & 41.3 & 41.0 & 17.8 & 4.5 & 64.0 \\
& 587 & 6.31 & 20.9 & 54.5 & 24.6 & 4.4 & 64.0 \\
Unit 4 & 597 & 5.19 & 43.7 & 41.0 & 15.3 & 3.5 & 64.4 \\
& 607 & 6.02 & 24.6 & 54.7 & 20.7 & 4.2 & 63.1 \\
& 617 & 5.94 & 26.9 & 53.4 & 19.8 & 2.9 & 65.8 \\
& 627 & 5.92 & 38.4 & 37.1 & 24.5 & 2.4 & 62.8 \\
& 637 & 6.09 & 13.0 & 70.0 & 17.0 & 2.9 & 62.0 \\
& 647 & 6.12 & 17.0 & 66.6 & 16.4 & 4.3 & 61.4 \\
& 657 & 5.65 & 20.9 & 67.3 & 11.8 & 3.7 & 62.6 \\
& 667 & 6.58 & 8.0 & 70.6 & 21.4 & 3.1 & 64.7 \\
& 677 & 4.41 & 58.7 & 33.9 & 7.4 & 4.3 & 65.6 \\
& 687 & 6.24 & 17.2 & 64.1 & 18.7 & 3.8 & 62.0 \\
& 697 & 6.81 & 3.0 & 73.0 & 24.0 & 4.8 & 61.8 \\
\hline
\end{tabular}




\begin{tabular}{|c|c|c|c|c|c|c|c|}
\hline \multicolumn{2}{|c|}{ Impositions (\%) } & \multicolumn{2}{|c|}{ Clay mineral ratios } & \multirow{2}{*}{ Unit } & \multirow{2}{*}{$\begin{array}{l}\text { depth } \\
\text { (cm) }\end{array}$} & \multirow[b]{2}{*}{${ }^{87} \mathrm{Sr} /{ }^{86} \mathrm{Sr}$} & \multirow[b]{2}{*}{$2 \sigma \mathrm{SE}$} \\
\hline Kaolinite & Chlorite & $\mathrm{K}+\mathrm{C}$ & $\mathrm{S} / \mathrm{I} * 100$ & & & & \\
\hline 11.7 & 20.1 & 31.8 & 2.4 & \multirow{3}{*}{ Unit 1} & 47 & 0.722591 & 0.000014 \\
\hline 10.7 & 19.9 & 30.6 & 1.9 & & 67 & 0.723603 & 0.000011 \\
\hline 10.5 & 19.1 & 29.6 & 2.6 & & 107 & 0.720661 & 0.000011 \\
\hline 11.0 & 18.5 & 29.5 & 2.9 & \multirow{2}{*}{ Unit 2-1 } & 137 & 0.723536 & 0.000017 \\
\hline 9.6 & 16.2 & 25.9 & 3.2 & & 197 & 0.719741 & 0.000011 \\
\hline 10.3 & 16.3 & 26.6 & 3.0 & Unit 2-2 & 277 & 0.719819 & 0.000014 \\
\hline 11.1 & 17.9 & 29.0 & 3.1 & \multirow{4}{*}{ Unit 3} & 327 & 0.718528 & 0.000027 \\
\hline 11.7 & 18.5 & 30.1 & 3.0 & & 397 & 0.720141 & 0.000007 \\
\hline 11.4 & 17.6 & 29.0 & 1.7 & & 447 & 0.720495 & 0.000050 \\
\hline 11.2 & 18.1 & 29.3 & 4.1 & & 517 & - & - \\
\hline 10.1 & 12.0 & 22.1 & 4.2 & \multirow{3}{*}{ Unit 4} & 567 & 0.720030 & 0.000008 \\
\hline 11.4 & 15.1 & 26.5 & 3.9 & & 627 & 0.720886 & 0.000008 \\
\hline 11.6 & 15.5 & 27.0 & 5.6 & & 697 & 0.719559 & 0.000008 \\
\hline 12.0 & 16.6 & 28.6 & 5.8 & & & & \\
\hline 12.5 & 13.9 & 26.4 & 6.5 & & & & \\
\hline 10.4 & 15.6 & 26.0 & 6.1 & & & & \\
\hline 11.1 & 16.7 & 27.8 & 9.5 & & & & \\
\hline 12.0 & 18.1 & 30.1 & 7.3 & & & & \\
\hline 11.8 & 17.2 & 29.0 & 7.1 & & & & \\
\hline 13.0 & 19.2 & 32.1 & 7.1 & & & & \\
\hline 12.7 & 18.5 & 31.2 & 7.9 & & & & \\
\hline 11.9 & 17.7 & 29.6 & 7.9 & & & & \\
\hline 13.0 & 20.0 & 33.0 & 5.7 & & & & \\
\hline 12.4 & 16.3 & 28.7 & 8.5 & & & & \\
\hline 13.2 & 15.9 & 29.1 & 10.5 & & & & \\
\hline 13.4 & 18.7 & 32.0 & 6.0 & & & & \\
\hline 14.2 & 18.1 & 32.3 & 7.1 & & & & \\
\hline 13.5 & 18.5 & 32.0 & 5.7 & & & & \\
\hline 13.3 & 18.7 & 32.1 & 7.5 & & & & \\
\hline 14.8 & 19.5 & 34.2 & 4.1 & & & & \\
\hline 14.5 & 20.0 & 34.4 & 6.7 & & & & \\
\hline 13.8 & 18.2 & 32.0 & 5.9 & & & & \\
\hline 13.5 & 18.0 & 31.6 & 6.5 & & & & \\
\hline 14.7 & 21.1 & 35.8 & 5.6 & & & & \\
\hline 14.2 & 19.0 & 33.2 & 5.9 & & & & \\
\hline 14.0 & 20.2 & 34.2 & 4.8 & & & & \\
\hline 13.5 & 20.2 & 33.7 & 4.1 & & & & \\
\hline 12.8 & 20.3 & 33.1 & 5.6 & & & & \\
\hline 14.0 & 18.7 & 32.7 & 5.4 & & & & \\
\hline 13.8 & 20.6 & 34.4 & 5.3 & & & & \\
\hline 13.1 & 20.1 & 33.2 & 5.4 & & & & \\
\hline 13.8 & 21.8 & 35.6 & 7.3 & & & & \\
\hline 14.3 & 19.8 & 34.1 & 5.8 & & & & \\
\hline 13.6 & 20.4 & 34.0 & 6.5 & & & & \\
\hline 13.7 & 20.2 & 33.9 & 5.6 & & & & \\
\hline 12.8 & 20.0 & 32.8 & 5.4 & & & & \\
\hline 12.3 & 20.6 & 32.8 & 6.7 & & & & \\
\hline 12.4 & 19.7 & 32.1 & 5.4 & & & & \\
\hline 14.0 & 20.2 & 34.2 & 4.5 & & & & \\
\hline 13.5 & 21.4 & 34.9 & 6.4 & & & & \\
\hline 13.6 & 20.9 & 34.4 & 5.2 & & & & \\
\hline 12.0 & 22.6 & 34.6 & 5.0 & & & & \\
\hline 12.7 & 19.6 & 32.3 & 5.9 & & & & \\
\hline
\end{tabular}




\begin{tabular}{llll}
12.5 & 18.4 & 31.0 & 7.7 \\
12.5 & 18.9 & 31.4 & 7.1 \\
13.4 & 18.2 & 31.6 & 6.9 \\
12.4 & 19.7 & 32.1 & 5.4 \\
12.7 & 20.0 & 32.7 & 6.6 \\
12.2 & 19.2 & 31.3 & 4.4 \\
13.6 & 21.2 & 34.8 & 3.9 \\
13.4 & 21.7 & 35.1 & 4.7 \\
14.0 & 20.3 & 34.3 & 6.9 \\
13.0 & 20.7 & 33.7 & 6.0 \\
12.7 & 19.5 & 32.1 & 4.9 \\
11.9 & 18.2 & 30.1 & 6.6 \\
13.0 & 21.2 & 34.2 & 6.1 \\
14.7 & 18.7 & 33.4 & 7.8 \\
\hline
\end{tabular}




\begin{tabular}{ccccccccc}
\hline $\begin{array}{c}\text { ir-Nd isotopes } \\
{ }^{143} \mathrm{Nd} /{ }^{144} \mathrm{Nd}\end{array}$ & $2 \sigma \mathrm{SE}$ & $\varepsilon \mathrm{Nd}$ & $\mathrm{SiO} 2$ & $\mathrm{Al}$ & $\mathrm{Al} 2 \mathrm{O} 3$ & $\mathrm{Fe} 2 \mathrm{O} 3(\mathrm{~T})$ & $\mathrm{MnO}$ & $\mathrm{MgO}$ \\
\hline \hline 0.511999 & 0.000004 & -12.47 & 56.35 & 8.13 & 15.36 & 5.83 & 0.051 & 2.85 \\
0.511988 & 0.000005 & -12.67 & 53.84 & 8.79 & 16.6 & 6.47 & 0.053 & 2.79 \\
0.511971 & 0.000004 & -13.00 & 54.54 & 8.22 & 15.54 & 6.09 & 0.053 & 2.66 \\
\hline 0.511880 & 0.000003 & -14.79 & 56.44 & 8.23 & 15.56 & 5.95 & 0.049 & 2.47 \\
0.511860 & 0.000003 & -15.17 & 65.44 & 6.87 & 12.99 & 4.6 & 0.043 & 1.75 \\
\hline 0.511908 & 0.000003 & -14.25 & 68.19 & 6.34 & 11.98 & 4.05 & 0.044 & 1.57 \\
\hline 0.512010 & 0.000003 & -12.25 & 62.59 & 7.15 & 13.51 & 5.05 & 0.069 & 1.94 \\
0.511980 & 0.000010 & -12.84 & 61.72 & 7.38 & 13.94 & 5.4 & 0.101 & 2.16 \\
0.511965 & 0.000007 & -13.12 & 61.55 & 7.14 & 13.49 & 5.17 & 0.104 & 2.1 \\
- & - & - & 62.98 & 7.18 & 13.56 & 5.03 & 0.079 & 2.08 \\
\hline 0.511883 & 0.000006 & -14.72 & 69.98 & 5.82 & 11 & 3.39 & 0.059 & 1.34 \\
0.511808 & 0.000010 & -16.19 & 65.73 & 6.41 & 12.12 & 4.15 & 0.073 & 1.71 \\
0.511817 & 0.000005 & -16.01 & 65.74 & 6.54 & 12.36 & 4.12 & 0.063 & 1.66 \\
\hline
\end{tabular}





\begin{tabular}{|c|c|c|c|c|c|c|c|c|}
\hline \multicolumn{9}{|c|}{ jor Elements (\%) } \\
\hline $\mathrm{CaO}$ & $\mathrm{Na} 2 \mathrm{O}$ & $\mathrm{K} 2 \mathrm{O}$ & $\mathrm{TiO} 2$ & $\mathrm{P} 2 \mathrm{O} 5$ & LOI & Total & $\mathrm{Sc}$ & $\mathrm{Be}$ \\
\hline 1.4 & 2.77 & 3.28 & 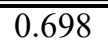 & 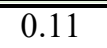 & 10.28 & 989.99 & 15 & 3 \\
\hline 1.88 & 2.64 & 3.37 & 0.757 & 0.12 & 10.7 & 99.21 & 15 & 3 \\
\hline 2.26 & 2.82 & 3.14 & 0.735 & 0.1 & 10.66 & 98.59 & 14 & 3 \\
\hline 1.53 & 2.68 & 3.08 & 0.754 & 0.08 & 10.63 & 99.22 & 14 & 3 \\
\hline 1.44 & 2.7 & 2.79 & 0.645 & 0.1 & 6.22 & 98.7 & 10 & 2 \\
\hline 1.96 & 2.71 & 2.75 & 0.607 & 0.09 & 5.05 & 99.02 & 9 & 2 \\
\hline 2.49 & 2.4 & 2.65 & 0.718 & 0.11 & 8.05 & 99.59 & 13 & 2 \\
\hline 3.03 & 2.38 & 2.81 & 0.702 & 0.12 & 7.92 & 100.3 & 13 & 2 \\
\hline 3.1 & 2.4 & 2.8 & 0.694 & 0.11 & 8.22 & 99.73 & 12 & 2 \\
\hline 3.03 & 2.39 & 2.71 & 0.724 & 0.09 & 7.53 & 100.2 & 12 & 2 \\
\hline 2.79 & 2.47 & 2.57 & 0.541 & 0.1 & 4.93 & 99.16 & 8 & 2 \\
\hline 3.59 & 2.34 & 2.69 & 0.623 & 0.11 & 6.42 & 99.54 & 10 & 2 \\
\hline 2.91 & 2.49 & 2.77 & 0.625 & 0.13 & 6.18 & 99.04 & 10 & 2 \\
\hline
\end{tabular}





\begin{tabular}{ccccccccc}
$\mathrm{V}$ & $\mathrm{Cr}$ & $\mathrm{Co}$ & $\mathrm{Ni}$ & $\mathrm{Cu}$ & $\mathrm{Zn}$ & $\mathrm{Ga}$ & $\mathrm{Ge}$ & $\mathrm{As}$ \\
\hline \hline 123 & 100 & 17 & 50 & 20 & 110 & 20 & 1.6 & 7 \\
126 & 110 & 17 & 40 & 30 & 120 & 22 & 1.6 & 6 \\
115 & 100 & 17 & 40 & 20 & 110 & 21 & 1.6 & 6 \\
\hline 113 & 100 & 17 & 40 & 20 & 100 & 20 & 1.4 & 6 \\
73 & 80 & 12 & 30 & 10 & 70 & 16 & 1.6 & $<5$ \\
\hline 62 & 70 & 10 & 30 & 10 & 60 & 15 & 1.4 & $<5$ \\
\hline 90 & 90 & 14 & 40 & 20 & 80 & 18 & 1.5 & 10 \\
92 & 90 & 14 & 40 & 30 & 80 & 18 & 1.5 & 8 \\
90 & 90 & 14 & 30 & 20 & 80 & 18 & 1.4 & 7 \\
85 & 80 & 14 & 30 & 20 & 80 & 17 & 1.4 & 5 \\
\hline 56 & 60 & 9 & 20 & 10 & 60 & 14 & 1.3 & 7 \\
72 & 80 & 11 & 30 & 20 & 70 & 16 & 1.5 & 7 \\
72 & 80 & 11 & 30 & 20 & 70 & 15 & 1.5 & 7 \\
\hline
\end{tabular}





\begin{tabular}{ccccccccc}
$\mathrm{Rb}$ & $\mathrm{Sr}$ & $\mathrm{Y}$ & $\mathrm{Zr}$ & $\mathrm{Nb}$ & $\mathrm{Mo}$ & $\mathrm{Ag}$ & $\mathrm{In}$ & $\mathrm{Sn}$ \\
\hline \hline 151 & 128 & 25.4 & 168 & 13.9 & $<2$ & 0.6 & $<0.1$ & 3 \\
162 & 139 & 25.7 & 156 & 13 & $<2$ & 0.6 & $<0.1$ & 3 \\
153 & 155 & 25.6 & 162 & 11.7 & $<2$ & 0.5 & $<0.1$ & 3 \\
\hline 151 & 137 & 25.9 & 168 & 12.9 & $<2$ & 0.5 & $<0.1$ & 3 \\
118 & 162 & 22.9 & 239 & 9.5 & $<2$ & 0.7 & $<0.1$ & 2 \\
\hline 109 & 184 & 23.2 & 251 & 8.7 & $<2$ & 0.7 & $<0.1$ & 2 \\
\hline 119 & 163 & 29.4 & 200 & 12 & $<2$ & 0.6 & $<0.1$ & 3 \\
125 & 180 & 27.1 & 174 & 10.8 & $<2$ & $<0.5$ & $<0.1$ & 3 \\
124 & 181 & 25.2 & 162 & 11.8 & $<2$ & $<0.5$ & $<0.1$ & 3 \\
118 & 186 & 25.4 & 179 & 10.6 & $<2$ & $<0.5$ & $<0.1$ & 3 \\
\hline 100 & 200 & 21.4 & 242 & 8.5 & $<2$ & 0.6 & $<0.1$ & 2 \\
110 & 205 & 27.2 & 273 & 9.4 & $<2$ & 0.7 & $<0.1$ & 2 \\
111 & 201 & 25 & 266 & 9.6 & $<2$ & 0.7 & $<0.1$ & 2 \\
\hline
\end{tabular}





\begin{tabular}{ccccccccc}
\hline \multicolumn{7}{c}{ Trace Elements $(\mathrm{ppm})$} \\
$\mathrm{Sb}$ & $\mathrm{Cs}$ & $\mathrm{Ba}$ & $\mathrm{La}$ & $\mathrm{Ce}$ & $\mathrm{Pr}$ & $\mathrm{Nd}$ & $\mathrm{Sm}$ & $\mathrm{Eu}$ \\
\hline \hline 1.1 & 10.7 & 457 & 40.6 & 81.7 & 9.1 & 33.7 & 6.46 & 1.38 \\
0.9 & 11.4 & 453 & 41.1 & 82.7 & 9.02 & 34.1 & 6.22 & 1.31 \\
0.9 & 10.5 & 432 & 42.1 & 84.7 & 9.23 & 34.3 & 6.67 & 1.32 \\
\hline 1.1 & 10 & 431 & 41.9 & 85.7 & 9.28 & 35.5 & 6.77 & 1.38 \\
0.7 & 6.3 & 479 & 37.6 & 75.2 & 8.26 & 31 & 5.99 & 1.12 \\
\hline 0.9 & 5.1 & 505 & 36.9 & 72.8 & 8.06 & 29.9 & 5.76 & 1.13 \\
\hline 1.1 & 7.6 & 473 & 40.9 & 82.5 & 9.09 & 33.6 & 6.89 & 1.35 \\
1.1 & 7.9 & 544 & 38.6 & 77.7 & 8.59 & 30.9 & 6.26 & 1.3 \\
1 & 7.5 & 550 & 36.2 & 73.8 & 8 & 29.7 & 5.97 & 1.26 \\
0.9 & 6.8 & 510 & 37.2 & 75.2 & 8.31 & 30.2 & 6 & 1.2 \\
\hline 0.9 & 4.1 & 564 & 34.6 & 69.2 & 7.74 & 27.6 & 5.5 & 1.07 \\
0.9 & 5.6 & 515 & 41.1 & 82.6 & 9.14 & 33.8 & 6.49 & 1.22 \\
1 & 5.4 & 595 & 40.3 & 79.2 & 8.84 & 31.9 & 6.34 & 1.22 \\
\hline
\end{tabular}





\begin{tabular}{ccccccccc}
$\mathrm{Gd}$ & $\mathrm{Tb}$ & $\mathrm{Dy}$ & $\mathrm{Ho}$ & $\mathrm{Er}$ & $\mathrm{Tm}$ & $\mathrm{Yb}$ & $\mathrm{Lu}$ & $\mathrm{Hf}$ \\
\hline \hline 5.4 & 0.83 & 4.93 & 0.92 & 2.69 & 0.421 & 2.74 & 0.402 & 4.6 \\
5.57 & 0.84 & 4.83 & 0.93 & 2.64 & 0.399 & 2.56 & 0.394 & 4.1 \\
5.5 & 0.86 & 4.8 & 0.92 & 2.65 & 0.375 & 2.62 & 0.395 & 4.1 \\
\hline 5.79 & 0.85 & 4.99 & 0.92 & 2.63 & 0.39 & 2.56 & 0.387 & 4.5 \\
4.95 & 0.75 & 4.19 & 0.82 & 2.31 & 0.346 & 2.29 & 0.368 & 5.9 \\
\hline 4.65 & 0.73 & 4.25 & 0.83 & 2.4 & 0.362 & 2.35 & 0.369 & 5.9 \\
\hline 5.66 & 0.88 & 5.29 & 1.03 & 3.06 & 0.459 & 2.98 & 0.44 & 5.3 \\
5.19 & 0.82 & 4.82 & 0.9 & 2.66 & 0.383 & 2.52 & 0.395 & 4.4 \\
5.14 & 0.77 & 4.4 & 0.86 & 2.62 & 0.368 & 2.47 & 0.384 & 4.2 \\
5.16 & 0.8 & 4.52 & 0.89 & 2.53 & 0.399 & 2.58 & 0.377 & 4.3 \\
\hline 4.76 & 0.69 & 3.89 & 0.77 & 2.27 & 0.334 & 2.16 & 0.336 & 5.5 \\
5.21 & 0.78 & 4.79 & 0.96 & 2.75 & 0.412 & 2.68 & 0.421 & 6.7 \\
5.31 & 0.77 & 4.4 & 0.87 & 2.55 & 0.386 & 2.49 & 0.365 & 6.4 \\
\hline
\end{tabular}





\begin{tabular}{ccccccc}
$\mathrm{Ta}$ & $\mathrm{W}$ & $\mathrm{Tl}$ & $\mathrm{Pb}$ & $\mathrm{Bi}$ & $\mathrm{Th}$ & $\mathrm{U}$ \\
\hline \hline 1.37 & 3.9 & 0.55 & 26 & $<0.1$ & 14.8 & 6.62 \\
1.41 & 3.2 & 0.52 & 23 & $<0.1$ & 15.3 & 6.81 \\
1.4 & 3.1 & 0.53 & 26 & $<0.1$ & 15.3 & 7.05 \\
\hline 1.43 & 3 & 0.57 & 22 & $<0.1$ & 15.2 & 7.89 \\
1.22 & 7.6 & 0.44 & 19 & $<0.1$ & 12.6 & 3.07 \\
\hline 1.16 & 2.6 & 0.44 & 18 & $<0.1$ & 11.8 & 2.24 \\
\hline 1.29 & 3.3 & 0.47 & 24 & $<0.1$ & 13.7 & 3.18 \\
1.29 & 3.2 & 0.48 & 21 & $<0.1$ & 13.3 & 2.75 \\
1.31 & 3 & 0.51 & 24 & $<0.1$ & 12.3 & 2.45 \\
1.27 & 5.2 & 0.48 & 20 & $<0.1$ & 12.3 & 2.25 \\
\hline 1.22 & 2.2 & 0.43 & 17 & 0.1 & 10.9 & 2.21 \\
1.15 & 3.2 & 0.45 & 20 & $<0.1$ & 13.9 & 2.6 \\
1.2 & 2.9 & 0.49 & 20 & $<0.1$ & 13.1 & 2.7 \\
\hline
\end{tabular}

